\title{
4 Die Entwicklung des „werbenden“ Films vor dem Ersten Weltkrieg
}

\subsection{Die Etablierung nationaler Produktionsfirmen}

Die Durchsetzung der festen Kinobetriebe brachte die Notwendigkeit mit sich, das Programm häufiger zu wechseln. ${ }^{141}$ Die Nachfrage nach Filmen, vor allem auch nach solchen, die dem lokalen Geschmack entsprachen, stieg stetig an und eröffnete heimischen Herstellern neue, Erfolg versprechende Möglichkeiten. Abseits der teils selbst produzierenden Wanderkinounternehmer hatten bereits ab 1906 einzelne kinematographisch Versierte sich in der Aufnahme von Filmen versucht. Während Josef Halbritter insbesonders Aktualitäten wie den Blumenkorso oder das Traberderby im Prater mit seiner Kamera festhielt, ${ }^{142}$ spezialisierte sich der gelernte Fotograf Johann Schwarzer auf „erotische Films“, die er über seine Firma „Saturn“ bis 1910 äußerst erfolgreich vertrieb. ${ }^{143}$

Auch zwei der namhaftesten österreichischen Filmpioniere starteten im Jahr 1906 erste, bescheiden ausfallende Filmversuche. ${ }^{144}$ Anton Kolm, der in Wien eine „fotografische Kunstanstalt“ betrieb, und sein Kameramann Jakob Fleck nahmen vorerst Firmungsbilder, Badeszenen im Gänsehäufel oder vorbeimarschierende Militärkapellen mit recht primitiven Apparaten in den Fokus. Für eine öffentliche Vorführung waren die Streifen aufgrund ihrer geringen Qualität nicht geeignet. ${ }^{145}$ Vier Jahre später hatten sich Kolm und Fleck jedoch bereits so umfassende kinematographische Fertigkeiten angeeignet, dass sie gemeinsam mit Louise Veltée-Kolm (spätere Fleck) die „Erste Österreichische Kinofilms-Industrie“ begründeten, die es sich - laut Pressemeldung zur Aufgabe machte, „aktuelle Ereignisse und Naturschönheiten unseres Vaterlandes kinematographisch wiederzugeben“. ${ }^{146}$ Ende 1910 wurde das Unternehmen zur

141 Die Autorin zitiert hier Kapitel 7 ihresArtikels: Moser,„Frühes Kino“, Kapitel 7 „Österreichische Filmpioniere“, http://ww1.habsburger.net/de/kapitel/oesterreichische-filmpioniere, 30.11.2014.

142 Fritz, Walter: Dokumentarfilme aus Österreich 1909-1914, Wien 1980, S. 15.

143 Im Februar 1911 wurde der weitere Vertrieb der Produktionen der Firma Saturn seitens des k.k. Landesgerichts Wien verboten. Vgl. dazu: Achenbach, Michael: Die Geschichte der Firma Saturn und ihre Auswirkungen auf die österreichische Filmzensur, in: Achenbach, Michael/Caneppele, Paolo/Kieninger, Ernst: Projektionen der Sehnsucht. Saturn. Die erotischen Anfänge der österreichischen Kinematografie, Wien 1999, S. 92-93.

144 Die Autorin zitiert hier Kapitel 7 ihres Artikels: Moser, „Frühes Kino“, Kapitel 7 „Österreichische Filmpioniere“, http://ww1.habsburger.net/de/kapitel/oesterreichische-filmpioniere, 30.11.2014.

145 Nepf, Markus: Die ersten Filmpioniere in Österreich. Die Aufbauarbeit von Anton Kolm, Louise Veltée/Kolm/Fleck und Jakob Fleck bis zu Beginn des Ersten Weltkriegs, in: Bono, Francesco/Caneppele, Paolo/Krenn, Günter: Elektrische Schatten. Beiträge zur österreichischen Stummfilmgeschichte, Wien 1999, S. 13. Fritz, Dokumentarfilme, S. 15.

146 Fritz, Dokumentarfilme, S. 16 bzw. Österreichischer Komet, Nr. 35, 8. Februar 1910, S. 5. 
„Österreichisch-Ungarischen Kinoindustrie“ umgewandelt, 1911 fand sich das Dreiergespann in einer neuen Produktionsfirma, der „Wiener Kunstfilm“, ein. ${ }^{147}$ Das Team Kolm-Veltée-Fleck war unter anderem darauf bedacht, sich am österreichischungarischen Markt zu profilieren, ein Gegengewicht zu den dominanten ausländischen Lieferanten zu bilden und im Aktualitätenbereich zu punkten, der bislang von französischen Unternehmen bedient wurde. ${ }^{148}$ Das Spektrum der von ihnen gedrehten Laufbilder war breit: kurze dokumentarische Streifen von aktueller und visuell ansprechender Art sowie Spielfilme, wobei soziale Dramen und Literaturverfilmungen letztlich ins Zentrum der Produktion rückten. In den Jahren 1910 bis 1911 produzierten Kolm und Fleck 48 dokumentarische Filme, darunter auch eine Reihe von Reisebildern, so etwa ÖsTERREICHISCHE ALPENBAHn, EINE FAHRT NACH MARIAZELL (1910), DER SEMMERING IM SCHNEE (1910), MÖDLING (1911), BADEN (1911). 1912 stellte die Gruppe Kolm-Velt ée-Fleck ihre dokumentarischen Aufnahmen ein, um sich fortan ausschließlich dem fiktionalen Film zu widmen. ${ }^{149}$

$\mathrm{Zu}$ Beginn der 1910er-Jahre wurden weitere, überwiegend kleinere Produktionsfirmen in Wien gegründet. Ihnen allen war gemein, dass sie „Naturaufnahmen“, im Speziellen Landschafts- und Reise-, aber mitunter auch Industriebilder in ihrem Programm hatten, ob sie sich nun „Duca-Film“, 150 „Dramagraph-Film“, 151 „Danubia“,", „W2 „Wiener Spezialfilm“, ${ }^{153}$ „Jupiter-Film“, 154 „Columbia Film-Fabrik“, 155

147 Bono, Francesco: Bemerkungen zur österreichischen Filmwirtschaft und Produktion zur Zeit des Stummfilms, in: Bono, Francesco/Caneppele, Paolo/Krenn, Günter: Elektrische Schatten. Beiträge zur österreichischen Stummfilmgeschichte, Wien 1999, S. 53-54.

148 Bislang hatten einzig französische Produzenten Drehgenehmigungen für offizielle Aufnahmen aus dem Kaiserhaus erhalten. So wurde, um nur ein Beispiel zu nennen, die Vermählung Erzherzog Karls mit Prinzessin Zita etwa von der Firma Gaumont gefilmt. Vgl. dazu auch: Nepf, Filmpioniere, S. 18 und 23.

149 Vgl. Fritz, Dokumentarfilme, Verzeichnis 1909-1914, Nr. 1-213. Nepf, Filmpioniere, S. 23. Bono, Bemerkungen zur österreichischen Filmwirtschaft, S. 54.

150 KITZBÜHEL (1914). Fritz, Dokumentarfilme, Verzeichnis 1909-1914, Nr. 139.

151 ABbaZia (1912), An Istriens GeSTAden (1912), Fritz, Dokumentarfilme, Verzeichnis 1909-1914, Nr. 83, 86.

152 WinteRVERGNÜGEN IN AUSSEE (1912), AUSSEE UND UMGEBUNG IM WinTER (1912), LANDWIRTSCHAFTLICHE MASCHINEN (1912), RAGUSA (1912). Fritz, Dokumentarfilme, Verzeichnis 1909-1914, Nr. 58, 60, 61, 63.

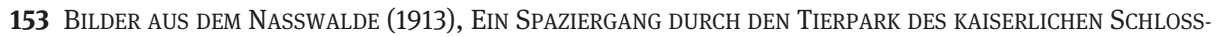
PARKES „SCHÖNBRUNN“ IN WIEN (1913), DIE HERSTELLUNG EINER ZIGARRE (1914). Fritz, Dokumentarfilme, Verzeichnis 1909-1914, Nr. 114, 117, 148.

154 DAS VerschwUndene Wien (1913), Der Rhein (1913), An ÖSTERREICHS AdRIA Teil I und II (1913). Als Kameramann der Jupiter-Film agierte Josef Halbritter. Siehe: Fritz, Dokumentarfilme, Verzeichnis 1909-1914, Nr. 106, 111, 119, 120.

155 Die von Josef Halbritter begründete „Columbia-Film“ kündigte sogar an, „Das Spezialfach werden Aufnahmen vaterländischer Natur- und Industriefilms bilden“. Beispiele dazu: EINE DAMPFSCHIFFAHRT DURCH DIE WACHAU (1913), Lunz AM SEE (1913). Fritz, Dokumentarfilme, Verzeichnis 1909-1914, Nr. 99, 101. Kinematographische Rundschau, Nr. 254, 19. Jänner 1913, S. 20. 
„Preiss-Film“, ${ }^{156}$ oder „Wiener Autorenfilm“157 nannten. Die Nachfrage nach Kurzfilmen (im Schnitt zwischen 80 und 160 Metern) hielt ungebrochen an. Das Publikum war an Langfilme von 1000 bis 2000 Metern noch nicht gewöhnt und beklagte bisweilen, obwohl die Gesamtvorstellung über eine Stunde dauerte, „zu wenig gesehen zu haben“. Die Besucher, besonders die in den äußeren Bezirken Wiens, verlangten nach mehr Abwechslung. ${ }^{158}$

Zum nationalen Hauptlieferanten dieser beliebten kurzen Laufbilder avancierte, nachdem sich Kolm-Veltée-Fleck aus dem dokumentarischen Bereich zurückgezogen hatten, Alexander „Sascha“ Joseph Graf Kolowrat-Krakowsky (kurz Sascha Kolowrat). Die von ihm 1910 im böhmischen Pfraumberg begründete „Sascha-Filmfabrik" konzentrierte sich in den Vorkriegsjahren auf Naturaufnahmen, Sportreportagen und Aktualitäten. ${ }^{159}$ In einer Anzeige der Firma des Jahres 1912 wies man auf das eigene Repertoire entsprechend hin: „Die ,Sascha'-Films dürften sich als Fabrikat erweisen, welches in keinem Kinematographentheater fehlen soll, denn die ,Sascha'-Filmfabrik hat sich zum Programm gemacht, nur interessante Naturaufnahmen herzustellen, wozu unser herrliches Vaterland so reichen Stoff bietet, ferner Industrieaufnahmen, die jedermann fesseln werden. “ ${ }^{\text {160 }}$ Die Fachpresse erwartete, dass die Verbindungen Kolowrats zur Aristokratie besonders ansprechende Sujets aus der heimischen Industrie nach sich ziehen würden. ${ }^{161}$ Der erste Industriefilm der „Sascha-Filmfabrik“, der auch der erste österreichischer Provenienz war, sollte diese Annahme bestätigen.

\subsection{Industriefilme und gewerbliche Filmpropaganda}

\footnotetext{
Kolossaler Erfolg bei der Probevorführung. DiE Gewinnung DES EISENS AM STEIRISCHEN ERZBERG IN EISENERZ. Glänzende Industrie-Aufnahme. Länge ca. 170 Meter. Ein hochinteressantes und landschaftlich schönes Bild. Prachtvolle Photographie. Wunderbare Virage. Ein unbestritten vollwertiges Fabrikat. Bestes vaterländisches Erzeugnis. ${ }^{162}$
}

156 WinTERPSORT IM ERZGEBIRGE (1914): Fritz, Dokumentarfilme, Verzeichnis 1909-1914, Nr. 131.

157 Von Toblach zum Misurinasee (1914), Am Wildbach (1914), Spalato (1914), StFit HeiligenkreuZ (1914), Durazzo, Die neue Residenz von Albanien (1914). Fritz, Dokumentarfilme, Verzeichnis 1909-1914, Nr. 137, 138, 142, 143, 153.

158 Österreichischer Komet, „Lange Films“, Nr. 71, 10. Juli 1911, S. 1, 5.

1591912 übersiedelte die Firma in die k.k. Residenzstadt, 1914 erfolgte ihre Registrierung im Wiener Handelsregister. Krenn, Günter: Der bewegte Mensch - Sascha Kolowrat, in: Bono, Francesco/Caneppele, Paolo/Krenn, Günter: Elektrische Schatten. Beiträge zur österreichischen Stummfilmgeschichte, Wien 1999, S. 38. Hübl, Maria: Sascha Kolowrat. Ein Beitrag zur Geschichte der Österreichischen Kinematographie, Dissertation, Wien 1950, S. 54-55.

160 Österreichischer Komet, „Anzeige ,Sascha’-Filmfabrik“, Nr. 87, 13. Jänner 1912, S. 23.

161 Hübl, Sascha Kolowrat, S. 29.

162 Kinematographische Rundschau, „Anzeige ,Sascha'-Filmfabrik“, Nr. 2020, 1912, S. 43. 
In derart euphorischer Manier wurde die erste österreichische Industriefilmproduktion begrüßt. Der „Werdeprozess der Erzgewinnung“, die sich „in großem Aufschwung befindet“, war Thema des Erzeugnisses. ${ }^{163}$ Dem Laufbild war ein großer Erfolg beschieden. 70 Kopien des Films wurden abgesetzt - eine Vertriebszahl, die eine österreichische Aufnahme bis zu diesem Zeitpunkt noch nie erzielen konnte. ${ }^{164}$ Zudem kam eine englischsprachige Version des Films in Umlauf. Das einzige heute überlieferte Material dieser Produktion basiert auf eben dieser Fassung.

A DAY AT AN AUSTRIAN IRON MinE (1912) präsentiert Ansichten des steirischen Erzberges sowie Spreng-, Abbau-, Transport- und erste Verarbeitungsprozesse vornehmlich in totalen und halbtotalen Einstellungen. Einzig eine Szene in der Innenanlage wurde - zur Veranschaulichung der Prüfung des liquiden Eisens durch die Bergarbeiter - in „American shot“ (Amerikanische) gedreht. Nah- und Detailansichten fehlen. Der Überblick über das Geschehen steht im Zentrum, wobei auf visuelle Effekte gesetzt wird: Bewegungsabläufe, Explosionen und Siedevorgänge entfalten ihre optische Wirkung. Berstendes Gestein, Staub- und Rauchschwaden, aufsteigende Dämpfe, gleißende Blitzlichter und Funken beleben das Bild und halten das zuschauende Auge in Bewegung. Anregende Panoramaarrangements verweisen auf geschulte Kameramänner.

Die einleitende Sequenz zur Demonstration der Beförderungsanlagen führt die Augen entlang der Förderschienen über eine Fluchtlinie in den Hintergrund, wo sich eine Wald- und Bergszenerie eröffnet. In der nachfolgenden Einstellung bildet die idyllisch erscheinende Landschaft gleichfalls ein Gegenstück zur modernen Waggon-Schiebevorrichtung, die im Vordergrund das Bild in Bewegung hält. Tradition und Moderne stehen permanent in visueller Konkurrenz. Per Dynamit erzeugte Explosionen stehen pittoresken Landstrichen gegenüber.

Althergebrachte Wahrnehmungsmuster werden aber mitunter noch bedient: Die Transportszenerie wird zeitlich wiederholt - die Aufwärtsbewegung der Erzzüge wird vorerst in einer Panoramaeinstellung und anschließend in totaler Ansicht präsentiert. Der räumlichen Einordnung der Szenerie folgt demnach eine genauere Beobachtung des Bewegungsablaufs.

A DAY AT AN AUSTRIAN IRON Mine verfolgte in Inhalt und Gestaltung zwei werberelevante Ziele: Einerseits propagierte der Film den hohen technischen und innovativen Standard des heimischen Eisenerzabbaus, andererseits suchte man gezielt attraktive landschaftliche Hintergrundmotive, um den touristischen Reiz der Region greifbar zu machen.

Im selben Jahr (1912) wurden weitere Industrie- und Lehrbilder österreichischer Provenienz fertiggestellt. Dr. Alto Arche, Lehrer an der Staatsrealschule XX., legte dem Ministerium für Unterricht und Kultus sechs Filme vor, die er in den Jahren

163 Österreichischer Komet, Nr. 85, 30. Dezember 1911. o. S. zit. nach: Hübl, Sascha Kolowrat, S. 30.

164 Guha, Wilhelm: Die Sascha-Filmfabrik, in Filmkunst, Nr. 74, 1976, S. 6-7. 
1907 bis 1912 für den Einsatz im Schulunterricht hergestellt hatte. Zur Umsetzung dieser Aufnahmen hatte ihm das Amt eine Subvention von 300 Kronen zukommen lassen. ${ }^{165}$ Bei dem Großteil der noch erhaltenen Streifen dieser Produktion handelt es sich um die Wiedergabe industriell-gewerblicher Arbeiten. ${ }^{166}$

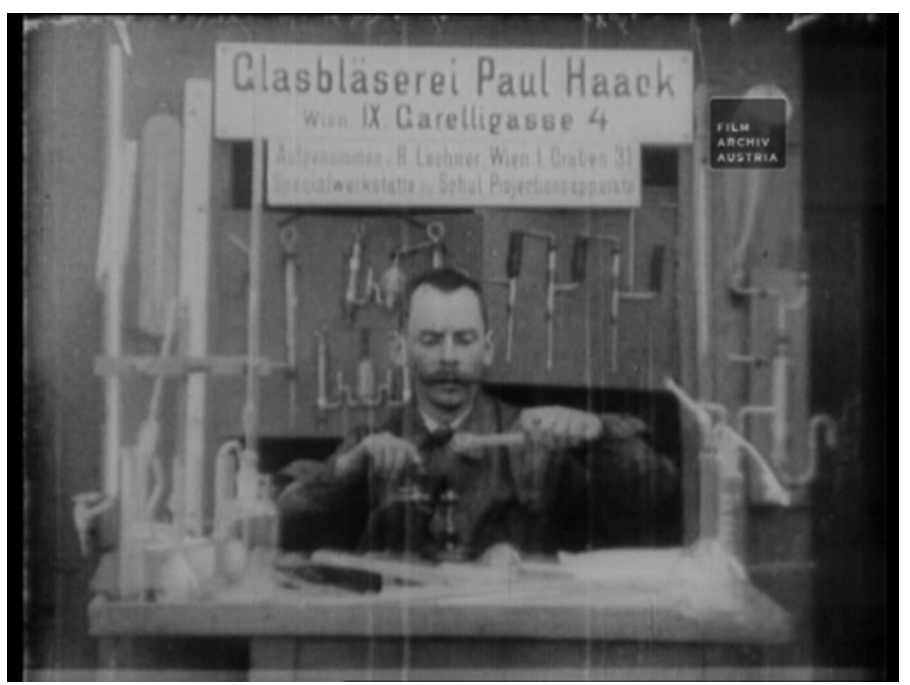

Abb. 1: GLASBLÄSEREI, A 1912.

Im Vergleich zur ersten Industriefilmproduktion der „Sascha-Filmfabrik“ fallen die Laufbilder Arches einfach aus. Handwerkliche Fertigungsprozesse wurden in einer einzelnen Kameraeinstellungen abgefilmt, wobei diese zwischen „Halbnah“ (GLASBLÄSER, 1912), „Amerikanisch“ (ZEUGFÄRBEREI, 1912) und „Halbtotal“ (HAFNER AN DER DREHSCHEIBE, 1912) variierten - je nachdem, ob die Darstellung eines Teils oder des gesamten Körpers bei der Verrichtung einer Tätigkeit von Relevanz war. Die Bearbeitung eines Werkstoffes (Glas erhitzen, ziehen, drehen, in Form biegen und blasen. / Flüssigkeiten vorbereiten, Kleidungsstücke tunken, wringen, aufhängen. / Lehm formen.) wird beobachtet. Erläuternde Zwischentitel fehlen gänzlich, die Bilder sind nicht selbsterklärend. Von einem erläuternden Kommentar bei der Aufführung des Gezeigten ist auszugehen, umso mehr, als die Filme für Unterrichtszwecke

165 Erlass Nr. 28074, 23. Juli 1907, Ministerium für Kultus und Unterricht, zit. nach Datenbankerfassung Filmarchiv Austria, Filmproduktion GLASBLÄSER (A 1912). Vgl. auch Fritz, Dokumentarfilme, S. 17. Fuchsig, Heinrich: Rund um den Film, Leipzig 1929, S. 124.

166 Einer der vier erhaltenen Filme zählt nicht zur Gattung Industriebild: KÜRTURNEN DER SCHÜLER DER K.K. FRANZ JoSEPH-REALSCHULE (A 1912). 
gedacht waren. Die Aufnahmen entsprechen dem Prinzip der „Ansicht“, es fehlt jegliches Narrativ, die Beobachteten (Handwerker) treten in direkten Kontakt mit der Kamera, sie sprechen, gestikulieren, verbeugen sich. Interessant ist, dass hier weniger für den einzelnen Betrieb geworben wird, ${ }^{167}$ sondern vielmehr die Produzenten der Laufbilder explizit im Titel genannt werden oder über Schilder, die während der gesamten Aufnahme zu sehen sind, ausgewiesen werden. Die Hersteller Dr. Alto Arche im Auftrag der Firma Lechner - warben demnach bei potenziellen Interessenten für ihre Gebrauchsfilmproduktion.

Das von Gunning bis etwa 1906 angesetzte „Kino der Attraktionen“"168 war demnach in Österreich-Ungarn in Hinblick auf den Industriefilm noch weit bis in die 1910er-Jahre gebräuchlich, während gleichzeitig Filme entstanden, die bereits eine zunehmend narrative Gestaltung verfolgten. $\mathrm{Zu}$ Letzteren zählten vor allem jene Produktionen, die für die öffentliche Aufführung gedacht waren. Die wenigen nachweisbaren industriell ausgerichteten Kurzfilmen der Vorkriegszeit hatten die Lampenproduktion, die Herstellung von Tabakwaren, den aktuellen technologischen Stand der Milchindustrie, landwirtschaftliche Maschinen oder die Filmproduktion selbst zum Thema. ${ }^{169}$

Früh ersichtlich war eine gewisse Verbindungslinie zwischen Industrie(werbe)und Lehrfilm, wie etwa auch die für den Unterricht vorgesehenen Streifen Alto Arches unter Beweis stellen. Schon 1907 wurde angesichts der Präsentation von Laufbildern an der Technischen Hochschule in Charlottenburg der doppelte Nutzen „technischer Filme“ in der Fachzeitung Der Komet beleuchtet. Einerseits könnten derartige kinematographische Bilder den Studierenden die Arbeitsweise komplexer Maschinen veranschaulichen, andererseits würden sie die Technik an sich nicht nur popularisieren, sondern sich zudem als eine zielgerichtete Reklame bei Fachausstellungen erweisen. ${ }^{170}$ Sieben Jahre später erkannte man in der Herstellung von Industriefilmen bereits einen national ökonomischen Vorteil. Ausgehend von britischen Beispielen betonte das Spezialmagazin des Produktions- und Verleihbetriebs „Eclair“ die Möglichkeiten, die diese Filme auch anderen Völkern und Staaten böten:

167 Einzig im Bild GLASBLÄSEREI wird das Unternehmen, in dem die Dreharbeiten stattfanden, ausgewiesen: Glasbläserei Paul Haack, Wien IX, Garellig. 4.

168 Gunning, Tom: The Cinema of Attractions, Its Spectator and the Avant-Garde, in: Wide Angle, Bd. 8, Nr. 3/4, 1986, S. 63-70.

169 Die FABRIKATION DER VERTEXLAMPE (A 1912, P: Wiener Kunstfilm-Industrie). Kinematographische Rundschau, Nr. 247, 1. Dezember 1912, S. 13. LANDWiRTSCHAFTLICHE MASChINEN (A 1912, P: Danubia). Mitteilungen der Österreichisch-Ungarischen Kinoindustrie Ges.m.b.H., Nr. 6, 10. Februar 1912, S. 3. Die Milch-Industrie in Prag (A 1913, P: unbekannt). Kino (Prag), 31. Oktober 1913. o. S. Die HerSTELLUNG EINER ZiGARRE (A 1914, P: Wiener Spezialfilm). Kinematographische Rundschau, Nr. 310, 15. Februar 1914, S. 76. Die IDEALE FilmERZEUGUnG (A 1914), P: Sascha-Filmfabrik. Fritz, Dokumentarfilme, Verzeichnis 1909-1914, Nr. 127.

170 Der Komet, „Der Kinematograph im Dienste technischer Belehrung“, Nr,. 1155, 1907, S. 9. 
„Ein kinematographisches Sammelwerk über die deutschen Industrien, über ihre Bedeutung, ihre sozialen Einrichtungen und ihre Ausbreitung über die Welt dürfte nach dreierlei Richtungen hin segensreich wirken. Es könnte die Achtung vor deutschem Industrie- und Gewerbefleiß im In- und Ausland erhöhen, Bildung, Belehrung und Anregung vermitteln und der Kinematographie eine neue ehrenvolle Rolle in unserem Kulturleben erringen." ${ }^{171}$ Wie sehr diese so früh formulierten Inhalte und Ziele des Industrie- und Kulturfilms in späteren Jahren noch von Relevanz waren, sollten die nachfolgenden Jahrzehnte zeigen. ${ }^{172} 1914$ ging die Filmkunst sogar so weit, die herkömmliche, humoristische Laufbildreklame als ermüdend und überholt darzustellen. „Viel mehr als diese belanglosen Zierlichkeiten“ interessiere „das Publikum von heute der schwere Ernst, der hinter dem allen steht, die Fabrikation, das Leben in der Fabrik“ ${ }^{173}$

In der Habsburgermonarchie, wo sich der werbende Film erst allmählich entwickeln und entfalten sollte, war man von derartigen Prognosen weit entfernt. Vielmehr entdeckten einzelne Branchen den reklametechnischen Wert der Kinematographie für sich, so etwa die Mode- und Textilindustrie. Vorbildfunktion hatte hier im Speziellen Frankreich. Pariser Modebilder zählten zum filmischen Repertoire diverser französischer Produktionsfirmen und fanden in ganz Europa Anklang. ${ }^{174} 1912$ entschloss sich der Wiener Modeklub, die Neuschöpfungen der großen Wiener Ateliers im Kinematographen vorzuführen. Der bisher gepflogene Widerstand der führenden Modehäuser, die vor allem Nachahmungen der eigenen Kreationen gefürchtet hatten, war gebrochen. Die Presse berichtete, dass nun erstmals im Koloriersaal des Modeschöpfers Arnold Bachwitz einem Fachpublikum „unter lebhaften Beifallsäußerungen“ Kostümbilder aus vergangener Zeit sowie Kleider der aktuellen Saison per Laufbild präsentiert wurden. ${ }^{175}$

Im darauffolgenden Jahr hatten die ersten Wiener Modefirmen bereits Verträge mit der Aktualitätenschau ECLAIR-REVUE abgeschlossen, denen zufolge ein kolorierter Wiener Modebericht regelmäßig in das Programm aufzunehmen war. ${ }^{176}$ Die Vorzüge dieser Form der Bewerbung akzentuierte man ausdrücklich: „In den plastischen Bildern des Kinematographen kommt der Reiz der neuen Modefarben schon deshalb besser zur Geltung, weil sie von lebenden Personen vor unseren Augen getragen werden. [...] Alles in allem ist also der Gesamteindruck weit eindringlicher als beim toten Modebild. Schnitt, Fall und Sitz des

171 Filmkunst, „Vom Reklamebild zur wissenschaftlichen Aufnahme“, Nr. 26, 26. Juni 1914, S. 1-2. 172 Siehe zu den Forderungen an den Industrie(werbe)film das Kapitel „Belehren - informieren werben: Forderungen an das Medium (Industrie-)Werbefilm“.

173 Filmkunst, „Vom Reklamebild zur wissenschaftlichen Aufnahme“, Nr. 25, 19. Juni 1914, S. 1-2.

174 Siehe z. B.: Österreichischer Komet, „Die Pariser Mode“, Nr. 76, 25. September 1911, S. 28.

175 Österreichischer Komet, „Die Mode im Kinematographen“, Nr. 107, 1. Juni 1912, S. 7.

176 Österreichischer Komet, „Wiener Mode in der Eclair-Revue“, Nr. 154, 26. April 1913, S. 9. 
Kleides, seine Wirkung als Ganzes, die so oft unberücksichtigt bleibt, kommen erst an dem lebenden Modelle zur Geltung." Hinsichtlich des zielgerichteten Einsatzes dieser neuen Werbemethode für die Bekleidungsindustrie zeigte man sich überzeugt: „Und da nun die Frauen den größten Teil des Kinopublikums stellen, so kann man mit Recht annehmen, dass der Kinematograph durch das lebende Modebild auf diese, als die eifrigsten Pflegerinnen aller Künste, eine neue Anziehungskraft ausüben wird. “177

Eine eigenwillig anmutende, visuell motivierte Reklameaktion unternahm die „Österreichische Hausindustrie“ 1914 im Rahmen ihres alljährlich stattfindenden Balles im Wiener Konzerthaus. In kinematographischen und „lebenden Visionsbildern“ warb man für die heimische Spitzenfabrikation. Der Abend begann mit der Vorführung des auf einer dalmatinischen Legende beruhenden Streifens DAS SPITZENWUNDER (1914). ${ }^{178}$ Der Plot gestaltete sich folgendermaßen: Der Fischer Epidauros lebt mit seiner schönen Tochter Ragusa in armseligen Verhältnissen. Dem erbarmungslosen Holzhändler Sergio schulden sie Geld, das dieser nun eintreiben will. Nur ein Eheversprechen Ragusas könnte ihn zum Einlenken bewegen. Doch diese liebt Dubrovnik, ${ }^{179}$ der gleichfalls mittellos ist und ihr von seinem Fischfang einzig eine seltsam geformte Alge mitbringt. In ihrer Verzweiflung betet Ragusa zur Muttergottes. Währenddessen schlingt sich Dubrovniks Wasserpflanze um das Gerippe des Fischernetzes und bildet ein apart erscheinendes Muster, welches das Mädchen aus Garn zur ersten Spitze nachahmt. Ihr Werk findet allseits Bewunderung und führt letztlich auch eine Märchenkönigin in die bescheidene Behausung. Voller Anerkennung überreicht sie Ragusa für ihre kunstreiche Arbeit einen Beutel mit Gold. Das Glück hat sich für alle eingestellt. „Seither“, so die Pressmeldung, „steht die Spitze unter dem besonderen Schutze königlicher Frauen. “180

Diesem sagenhaft konzipierten Reklamefilm folgte vor Ort die pantomimische Darstellung historisch motivierter Szenen unter dem Motto „Der Spitze Siegeslauf“, arrangiert von Dr. Gregor von Pantasi. Begleitet wurde die Präsentation von einer kroatischen Tamburizza-Kapelle. Nach venezianischen, flämischen und französischen Tableaus folgte das „Huldigungsbild Österreichische Spitzen“: „Aus der Mitte des Bühnenhintergrundes erhebt sich die Büste Sr. Majestät des Kaisers, über die

177 Österreichischer Komet, „Die Wiener Mode in der Eclair-Revue“, Nr. 155, 3. Mai 1913, S. 8-9.

178 DAs SPITZEnwunder, A 1914, P: Duca-Film (Wien), Regie (R): Fritz Freund, Albert Schloß, Drehbuch (DB): Lo Wilsdorf, Darsteller (D): Lucie Freifrau von Morpurgo-Lavall, Branimir Oršegić, Iphigenie Buchmann, Lucy Kieselhausen.

179 „Dubrovnik“ (kroatisch) und „Ragusa“ (italienisch) bezeichnen dieselbe Stadt im heutigen Kroatien. Hier tragen zwei Liebenden diese Stadtnamen.

180 Reichspost, „Das Spitzenwunder. Vorspiel zum Hausindustrieball“, 18. Februar 1914, S. 9. Wiener Zeitung, „Das Spitzenwunder“, 18. Februar 1914, S. 3. 
der Doppeladler seine Schwingen ausbreitet. Die von sämtlichen Damen des Großen Aktionskomitees ${ }^{181}$ dargestellten Völker Österreichs, mit den kostbarsten Spitzen österreichischer Provenienz beladen, huldigen dem geliebten Monarchen und reichen Ihm [sic!] den Lorbeer. Während unter den Klängen der Volkshymne der Vorhang von diesem prächtigen Bilde gezogen wurde, erhoben sich die den Saal füllenden Zuschauer spontan von ihren Sitzen und brachen in Begeisterung aus. “182 Der von k. k. Pathos getragene Akt zur Popularisierung der österreichischen Spitzenindustrie fand unter Beteiligung des Hofes, des Adels, der Industrie und unter der Patronanz der Erzherzogin Maria Josepha statt. ${ }^{183}$ Das gemeinsame Auftreten und Wirken von Staat und Wirtschaft unter Zuhilfenahme werbewirksamer kinematographischer Mittel hatte seinen Anfang genommen. Noch intensiver war diese Form der kooperativen Propaganda allerdings im Bereich des Fremdenverkehrs.

\title{
4.3 Reise- und Tourismusfilme
}

\begin{abstract}
Die Aufnahmen aus unbekannten Gebieten, die Vorführung von Naturwundern, die das Auge vielleicht nie geschaut hätte, von Sitten und Gebräuchen, von Menschen, mit denen der Nichtgereiste vielleicht nie im Leben Bekanntschaft machen würde, die Wiedergabe ihrer Trachten usw., das alles bildet eine lebende Reklame für fremde Länderstriche und zugleich die beste Reklame, da sie die Kinematographie direkt in den Dienst des Fremdenverkehrs stellt. ${ }^{184}$
\end{abstract}

Die von der Fachzeitung Österreichischer Komet skizzierte ökonomisch-touristische Wirkungskraft der verschiedenartige Regionen und Volkskulturen in den Fokus nehmenden „Naturaufnahmen“ hatten internationale Produktionsfirmen bereits erkannt. Kamerateams der großen kinematographischen Gesellschaften waren seit Jahren auf Reisen, um sehenswerte Gegenden und außergewöhnliche, optisch ansprechende Brauchtumsszenerien auf Zelluloid zu bannen. Auch auf dem Gebiet der Habsburgermonarchie fanden sich zahlreiche Motive, die von vornehmlich

181 Zum Aktionskomitee zählten Gräfin George Hoyos, Helene Löbl, Anna Schleif von Degenwart, Alina Römer-Perathoner, Lo Wilsdorf, Direktor Rudolf Ethofer, Fritz Freund, Dr. Fritz Minkus, Dr. Gregor von Pantasi und Albert Schloß. Neues Wiener Tagblatt, „Die Geburt der Spitze“, 18. Februar 1914, S. 14-15.

182 Wiener Zeitung, „Das Spitzenwunder“, 18. Februar 1914, S. 4. Die Zeit, „Der Hausindustrieball“, 17. Februar 1914, S. 3. Wiener Allgemeine Zeitung, „Der Hausindustrieball“, 18. Februar 1914, S. 2.

183 Als Vertreter des Kaisers erschien Erzherzog Karl Franz Josef, der spätere Kaiser Karl I und Sohn der Protektorin des Verbandes der Spitzenindustrie, Erzherzogin Maria Josepha. Wiener Allgemeine Zeitung, „Der Hausindustrieball“, 18. Februar 1914, S. 2. Im Rahmen der Veranstaltung erklärte der Erzherzog zudem, dass er ein „warmer Freund der Kinematographie“ sei. Vgl. Die Filmwoche, „Das Spitzenwunder“, Nr. 50, 1914, S. 52.

184 Österreichischer Komet, „Die Zukunft der Naturaufnahmen“, Nr. 115, 27. Juli 1912, S. 1. 
französischen Produzenten gefilmt und in den weltweiten Vertrieb übernommen wurden. „Éclipse“ hatte etwa die Reisebilder VON TRIEST NACH PORTOROSE UND MIRAMARE (F 1911), EINE FAHRT MIT DER MENDELBAHN (F 1912), BildER AUS MÄHREN (F 1912), ÖSTERREICHS BALKANLÄNDER: BOSNIEN (F 1912) oder ÖSTERREICHISCHE KÜSTE AN DER ADRIA (F 1912) im Programm. ${ }^{185}$ „Gaumont“ vertrieb gleichfalls k.k. Naturaufnahmen, wie u. a. AN DER DONAU SCHÖNEM STRANDE (BUDAPEST) (F 1910), DIE MALERISCHE BRÜHL (F 1910), ZELL AM SEE (F 1911), QUER DURCH WiEN (F 1911), ANSICHT VON ZARA (DALMATIEN) (F 1912), Winter in Galizien (F 1912) und Die SAlzBuRger SeEn (F 1913). ${ }^{186}$ „Pathé“ führte nicht nur DIE ÖSTERREICHISCHEN ALPEN IM BILDE im Repertoire, ${ }^{187}$ sondern wurde zudem vom „Österreichischen Skiverein“ mit der Herstellung einer Reihe von Wintersportbildern beauftragt. ${ }^{188}$ Dies entsprach einem sich langsam durchsetzenden, neuen Auftragsschema. Tourismusverbände, Städte und Gemeinden ließen optisch attraktive Landschafts- und Ortsfilme produzieren, um zugleich mit den Auftragnehmern Verträge über den breitenwirksamen Vertrieb der Laufbilder abzuschließen. ${ }^{189}$

In Österreich-Ungarn zeigten staatliche Behörden vermehrt Interesse an der kinematographischen Fremdenverkehrspropaganda. ${ }^{190}$ Schon 1911 hatte das österreichische Arbeitsministerium „zwei höhere Beamte zu diesem Zwecke auf Reisen geschickt“, die, mit kinematographischem Bildmaterial ausgestattet, in allen großen Städten Europas Vorträge hielten. Unter den mitgeführten Filmen fanden sich Aufnahmen aus Tirol, Salzburg und der Oststeiermark. Weitere kinematographische Landschaftsbilder österreichischer Herkunft wurden großen deutschen Kinobetreibern kostenfrei zur Verfügung gestellt. Laut Pressemeldung war geplant, an erster Stelle auch filmische Reklame für die Residenzstadt zu betreiben. Die ,schönsten Punkte Wiens und seiner näheren Umgebung“ sollten kinematographisch festgehalten werden. Zugleich hätten die Laufbilder „das gesellschaftliche Leben, das geschäftliche Treiben, Einkaufsmöglichkeiten und alle Genüsse des Körpers und Geistes“, welche die Kaiserstadt bietet, stimmig zu vermitteln. ${ }^{191}$

185 Das Lichtbild-Theater, Nr. 19, 7. Dezember 1911, S. 12. Ebd., Nr. 7, 15. Februar 1912, S. 11.Ebd., Nr. 14, 4. April 1912, S. 15-16. Ebd., Nr. 55, 29. August 1912, S. 12-13. Ebd., Nr. 42, 17. Oktober 1912, S. 24-25.

186 Kinematographische Wochenschau, Nr. 26, 1910, S. 16. Ebd., Nr. 36, 1910, S. 13. Ebd., Nr. 8, 1911, S. 22. Ebd., Nr. 44, 1911, S. 18-19. Ebd., Nr. 31, 1912, S. 27. Ebd., Nr. 47, 1912, S. 37. Ebd., Nr. 6, 1912, S. 27 f.

187 Kinematographische Rundschau, Nr. 320, 1914, S. 64.

188 Österreichischer Komet, „Kinematographische Wintersportbilder“, Nr. 95, 9. März 1912, S. 8.

189 Vgl. dazu etwa: Österreichischer Komet, „Die Zukunft der Naturaufnahmen“, Nr. 115, 27. Juli 1912, S. 3.

190 Die Autorin zitiert hier Kapitel 4 ihres Artikels: Moser, Filmischer Aufbruch in die Moderne, Kapitel 4 „Vom Fahren und Reisen: Fremdenverkehr und Tourismusfilme“, http://ww1.habsburger. net/de/kapitel/vom-fahren-und-reisen-fremdenverkehr-und-tourismusfilme, 30.11.2014.

191 Ebd., S. $3 \mathrm{f}$. 
Wenige Monate vor Bekanntmachung der weiteren staatlichen Pläne im Bereich der Tourismusförderung war die „Sascha-Filmfabrik“ bereits aktiv geworden und hatte ihre ersten sieben Filme, welche „die prächtigen Naturschönheiten Österreichs“ präsentierten und der Hebung des Fremdenverkehrs dienen sollten, dem Ministerium für öffentliche Arbeiten zur Ansicht vorgelegt. Offensichtlich erhoffte sich die neue österreichische Produktionsfirma über diese Eigenwerbung künftige behördliche Aufträge. Das k.k. Ministerium äußerte sich lobend über die vorgeführten Bilder, ${ }^{192}$ die ersten nachfolgenden, staatlich initiierten Filmprojekte wurden jedoch an ein französisches Unternehmen vergeben.

Die Serie „Österreich im lebenden Bilde“, produziert von der Wiener Zweigstelle der Firma „Eclair“, der „Wiener-Autorenfilm“, warb in den folgenden Jahren weltweit für den Besuch der heimischen Regionen. ${ }^{193}$ Mit arglos naiver Euphorie wurde diese fortschrittlichste Form der Tourismusreklame begrüßt: „Vorträge werden nicht besucht, die gewöhnliche Photographie ist der beweglichen unterlegen, und das Kino ist der Allerweltsfreund, der moderne Agitator, der Instruktor par excellence. Das Kino fälscht nicht, es erleidet keine Retuschen, es lässt sich nicht vom Lokalpatriotismus beeinflussen, es ist der moderne Wahrheitsfanatiker. “194

Im Zentrum der von der k.k. priv. Südbahn-Gesellschaft (Wien) und dem Arbeitsministerium beauftragten Filmreihe standen das Adriagebiet und die Alpenwelt. Mitgliedern des Kaiserhauses und den Spitzen der Regierungsbehörden wurden erstmals Aufnahmen aus dieser Serie im Rahmen einer Aufführung in der Wiener Urania vorgeführt. Zur Präsentation kamen „malerische Szenen“ der Adria, aufgenommen vom Bord des Lloyddampfers „Prinz Hohenlohe“ (Seebad Lovrana, Insel Arbe, Spalato und Ragusa an der dalmatinischen Küste). Es folgte eine Reise durch die Dolomiten, die „von Toblach mit seinem herrlichen See an der Croda Rossa und den Cadinispitzen vorbei nach Misurina, einer Perle der Alpenseen“, führte. Ein weiteres Laufbild widmete sich dem Kurort Abbazia, woran sich eine Reihe von Bildern aus San Martino di Castrozza schloss. ${ }^{195}$

192 Österreichischer Komet, „Hohe Anerkennung für Sascha-Films“, Nr. 90, 3. Februar 1912, S. 21 f. Die Produktionen der Sascha-Film wurden zudem im Oktober 1912 im Zuge der „Internationalen Kino-Ausstellung“, der ersten Gesamtschau des österreichischen Kino- und Filmwesens, ausgezeichnet. Vgl. dazu Guha, Sascha-Filmfabrik, S. 7.

193 Die Autorin zitiert hier Kapitel 4 ihres Artikels: Moser, Filmischer Aufbruch in die Moderne, Kapitel 4 „Vom Fahren und Reisen: Fremdenverkehr und Tourismusfilme“, http://ww1.habsburger. net/de/kapitel/vom-fahren-und-reisen-fremdenverkehr-und-tourismusfilme, 30.11.2014.

194 Österreichischer Komet, „Die Eclair-Films als Mittel zur Hebung des Fremdenverkehrs“, Nr. 177, 4. Oktober 1913, S. 36.

195 Filmkunst, „Österreich im lebenden Bilde und die Wiener Presse“, Nr. 45, 7. November 1913, S. 3-5. Filmkunst, „Das Bild aus Österreich“, Nr. 42, 17. Oktober 1913, S. 1-2. Deutsches Volksblatt, „Der Film als Fremdenverkehrsförderer“, 30. September 1913, S. 6-7. Als Einzeltitel konnten folgende Filme identifiziert werden: Von Toblach zum MisuRinaSEe (Dolomiten) (F/A 1914). Siehe: Filmkunst, Nr. 9, 27. Februar 1914, S. 12. SAN MARTINO DI CASTROZZA (SÜDTIROL) (F/A 1913). Vgl.: Filmkunst, 
Zwei Filme der Produktionsreihe erregten besondere Aufmerksamkeit und wurden mit regem Beifall bedacht. ${ }^{196}$ Das Außergewöhnliche an ihnen war, dass sie burleske Episoden vor dem Hintergrund interessanter landschaftlicher Szenerien darboten. Die komische und mitunter dramatische Handlung stand also im Mittelpunkt des Geschehens, die „Naturschönheiten Österreichs“ boten dazu den pittoresken Rahmen. Der Film Evas RosEngaRTENTOUR (1913) ${ }^{197}$ führt eine junge Amerikanerin von Wien nach Südtirol. An der Seite eines schneidigen Bergführers passiert sie gefährliche Felsen, lernt Volk und Brauchtum kennen, bestaunt die Grödener Tracht wie auch die regionale Holzschnitzkunst. Letztlich begeistert sie sich nicht nur für die mannigfaltige Pracht der Dolomiten, sondern auch für ihren Begleiter. ${ }^{198}$

In der Produktion ZWISCHEN ZWEI FEUERN (1913) ${ }^{199}$ wird der Lebemann Gottlieb von seinem Arzt zu einem Ruheaufenthalt auf dem Semmering angehalten. Auf seinen Spaziergängen begegnet der Filou jeweils einer brünetten und einer blonden Dame, die er beide fortan umschwärmt. Seinen Vorsätzen zum Trotz geht er seinem lukullischen und libidinösen Drang gleichermaßen ungebändigt nach. Die beiden jungen Frauen schreiben indessen ihren Ehemännern von den Zudringlichkeiten Gottliebs, was zur Anreise der Gatten und der nachfolgenden Flucht des vermeintlichen Frauenverstehers führt. ${ }^{200}$ Im Verlauf der Humoreske „lernt das Publikum fast das ganze Semmeringgebiet, alle die lieblichen Ausblicke von oben, den Sonnwendstein, kurzum alles das, was man in einem gewöhnlichen Ansichtsalbum nicht in zwei Stunden durchsehen könnte, kennen“. ${ }^{201}$ Die geschickt gewählte, mit witzigen und bekannten Stücken versehene musikalische Begleitung verstärkte

Nr. 42, 17. Oktober 1913, S. 2-3. HeILIGENKREUZ (F/A 1913). Vgl.: Filmkunst, Nr. 50, 12. Dezember 1913, S. 19. Spalato (F/A 1913). Siehe: Fritz, Dokumentarfilme, Verzeichnis 1909-1914, Nr. 142.

196 Die Autorin zitiert hier Kapitel 4 ihres Artikels: Moser, Filmischer Aufbruch in die Moderne, Kapitel 4 „Vom Fahren und Reisen: Fremdenverkehr und Tourismusfilme“, http://ww1.habsburger. net/de/kapitel/vom-fahren-und-reisen-fremdenverkehr-und-tourismusfilme, 30.11.2014.

197 Evas Rosengartentour, F/A 1913, P: Wiener Autorenfilm (Eclair, Französische Film- und Kinematographengesellschaft m.b.H. Wien, D: Eva Roth, Egon Friedell, Ivy Farren. Vgl.: Thaller, Anton (Hg.): Die österreichische Filmografie, Bd. 1, Spielfilme 1906-1918, Wien 2010, S. 11.

198 Ebd. Sowie Neue Freie Presse, „Österreich im Kino. Der Film für den Fremdenverkehr“, 30. September 1913, S. 10. Österreichischer Komet, „Die Eclair-Films als Mittel zur Hebung des Fremdenverkehrs“, Nr. 177, 4. Oktober 1913, S. 36. Österreichischer Komet, „Evas im Rosengarten“, Nr. 370, 16. Juni 1917, S. 32. Die Filmwoche, „Der Film als Fremdenverkehrsförderer“, Nr. 30, 5. Oktober 1913, S. 24.

199 ZWISCHEN ZWEI FEUERN, F/A 1913, P: Wiener Autorenfilm (Eclair, Französische Film- und Kinematographengesellschaft m.b.H. Wien, DB: Felix Fischer, D: Julius Brandt, Camilla von Nagy, Else Schreiber, Max Ralf-Ostermann, Max Falkenberg. Vgl.: Thaller, Filmografie, S. 199-201.

200 Ebd. Sowie Filmkunst, Nr. 12, 20. März 1914, S. 4 f.

201 Österreichischer Komet, „Die Eclair-Films als Mittel zur Hebung des Fremdenverkehrs“, Nr. 177, 4. Oktober 1913, S. 36. 
die suggestive Kraft der humoristischen Aufnahmen. ${ }^{202}$ Die Andersartigkeit der präsentierten Reklamefilme wurde als besonders einfallsreich und wirkungsvoll wahrgenommen: „Wohl die glücklichste Bereicherung des Gedankens war es übrigens auch die Psychologie des Kinopublikums in Rechnung zu ziehen und ihm nicht bloß die Herrlichkeiten der Natur vorzuführen, sondern ihm auch Amüsement zu bieten. “203

Eine andere Form der Dramatisierung wählte die „Hida-Film“, die 1914 als erste österreichische Produktionsfirma gleichfalls von der k.k. priv. Südbahn-Gesellschaft (Wien) und im Übereinkommen mit dem k.k. Eisenbahnministerium sowie dem k.k. Ministerium für öffentliche Arbeit beauftragt wurde, heimische „Naturaufnahmen mit belebten Szenerien“ zu Propagandazwecken „in origineller Weise“ in Verbindung zu bringen. ${ }^{204}$ Der unter diesen Vorgaben entstandene Film UNTER PALMEN UND EWIGEM EIS (1914) ${ }^{205}$ behandelt eine Dreiecksbeziehung, die in Ägypten, Abbazia und in der Gletscherwelt Tirols ihren Verlauf nimmt. Ein junges Mädchen, Vera, nimmt auf Drängen ihrer Eltern den erheblich älteren, aber reichen Fabrikanten Valimer zum Mann. Das Paar bereist Ägypten, als dringende geschäftliche Belange Valimer nach Hause rufen. In Abbazia wird Zwischenstation gemacht. Vera verbleibt dort mir ihrer Freundin Frau Holm, während ihr Gatte abreist. In dessen Abwesenheit lernt die junge Ehefrau den Sportsmann und Grafen Henry kennen. Sie verlieben sich und reisen weiter in die Tiroler Berge. Indessen erkennt Valimer, dass er durch das Fehlverhalten anderer seinen Bankrott erklären muss, in der Folge erkrankt er schwer. Vera erhält Nachricht von den letzten Ereignissen, bleibt jedoch in Tirol. Im Zuge eines Skiausflugs wird Graf Henry von einer Lawine erfasst und stirbt. Vera findet seinen reglosen Körper und erfriert selbst in der verschneiten, kalten Winterlandschaft. ${ }^{206}$

Obwohl die Presse den larmoyanten Plot des Films mitunter kritisierte, zeigte sie sich andererseits von den in modernster Technik umgesetzten Landschafts-, Meeresbildern und Wintersportaufnahmen beeindruckt: „Fachleute, die der gestrigen Premiere des Films beiwohnten, waren einig, dass in Österreich bisher noch niemals auch nur annähernd so schöne Filmaufnahmen zustande gebracht wurden. Tatsächlich hörte man während der Vorführung immer wieder Rufe des Entzückens“. ${ }^{207}$ Als besonders durchdacht empfand man den Umstand, dass es sich hier

202 Filmkunst, „Was die Presse sagt!“, Nr. 11, 13. März 1914, S. 15. Südbahn und Llyod, „Der Film als Fremdenverkehrsförderer“, Nr. 11, 1. November 1913, S. 33.

203 Kinematographische Rundschau, „Österreich im Film“, Nr. 291, 5. Oktober 1913, S. 109.

204 Die Filmwoche, „Der Film als Fremdenverkehrsförderer“, Nr. 60, 3. Mai 1914, S. 35.

205 Unter Palmen und EWigem EIS, F/A 1914, P: Hida-Film (Cornelius Hintner/Richard Damaschka, Wien), DB: Cornelius Hintner, D: Grete Huth, Emanuel Feuereisen. Vgl.: Thaller, Filmografie, S. 193-196.

206 Ebd. sowie Die Filmwoche, „Eine österreichische Filmsensation“, Nr. 61, 10. Mai 1914, S. 2-5. 207 Neue Kino-Rundschau, Nr. 42, 22. Dezember 1917, S. 74-75. Neue Freie Presse, Abendblatt, Die Filmwoche, „Der Film als Fremdenverkehrsförderer“, Nr. 60, 3. Mai 1914, S. 35. 
vor allem um eine „unauffällige Form der Reklame“ handle. Es sei eine nennenswerte Leistung einiger Unternehmer - folgerte die Arbeiter-Zeitung (!) -, „dass sie den Film in den Dienst der Industrie oder des Fremdenverkehrs stellt“, ohne dass der Großteil des Publikums ahnt, dass man es für eine Sache zu gewinnen versucht. „Man merkt kaum, dass die prachtvollen Wandelbilder von Landschaftsschönheiten Selbstzweck waren, so sehr verflochten sie sich mit der Handlung.“ ‘208

Wie „dezent“ die Reisebilder aus Österreich-Ungarn dieser Jahre hinsichtlich ihrer Werbewirkung tatsächlich ausfielen, lässt sich in den meisten Fällen nicht mehr eruieren. Die Mehrheit dieser Aufnahmen gilt als verschollen. Eines der wenigen erhaltenen Beispiele aus der Produktion der „Österreichisch-Ungarischen Kinoindustrie“, MARIAZELL (1911), ist noch stark vom „Ansichtscharakter“ geprägt. Es fehlen ersichtliche und narrativ richtungsweisende Anfangs- und Endpunkte. ${ }^{209}$ Szenerien in totaler Einstellung und ebenerdiger Kameraposition sind aneinandergereiht. Straßenzüge, Plätze werden abgeschwenkt, eine Übersicht über Bauwerke (z. B. Haus „Zum Guten Hirten“, Basilika Mariazell usw.) und Landschaftsansichten wird geboten. Von einem erhöhten Standpunkt aus wird der Hauptplatz der Stadt allmählich Punkt für Punkt gestreift, das teils getönte Material verweist auf eine den Ausdruck nuancierende Viragierung. Den Innenansichten Mariazells folgen Bilder, die von außen einen Blick auf die Stadtanlage und ihre landschaftlich idyllische, gefällige Umgebung gewähren. Die mehr oder weniger offensichtliche Präsentation von Gästehäusern des Orts (Hotel Goldener Löwe, Hotel Goldenes Kreuz) lässt vermuten, dass hier im Hintergrund durchaus auch Auftraggeber fungierten. ${ }^{210}$ Der Absatz derartig gestalteter Bilder schien grundsätzlich garantiert. So wurden dem Fremdenverkehr dienende Filme ,jederzeit bereitwillig“ von Kinounternehmern „gegen einen bloßen Spesenersatz“ im Programm platziert. ${ }^{211}$

$\mathrm{Zu}$ den zentralen Herstellern österreichischer Travelogues vor dem Ausbruch des Ersten Weltkriegs zählte bis inklusive 1912 das Team Kolm-Veltée-Fleck, danach dominierte Sascha Kolowrat diesen Bereich der Laufbildproduktion. Die Reisebilder aus der Monarchie waren vielfältig: Brachte die „ÖsterreichischUngarische Kinoindustrie“ etwa PRAG (1911), GRAZ (1911), MELK (1911), LAXENBURG (1911), Kroatische SeEn (1911), Das Thayatal (1911) oder DAs InNTAL (1911) ${ }^{212}$ in die

208 Arbeiter-Zeitung, „Film und Fremdenverkehr“, 12. Mai 1914, S. 6.

209 Das Fehlen von Anfangs- und Endmarkern ist durchaus charakteristisch für die fiktionalen Ansichten der kinematographischen Frühzeit. Vgl. dazu auch: Jung, Ästhetischer Wandel, S. 225.

210 Reisefilme waren in den meisten Fällen Auftragsfilme. Die Sichtbarmachung von Transportunternehmen oder Hotels ist oft als indirekter Hinweis auf die Financiers der Produktion zu verstehen. Vgl. dazu: Jacques, Pierre-Emmanuel: Reise- und Tourismusfilme, in: Zimmermann, Yvonne (Hg.): Schaufenster Schweiz. Dokumentarische Gebrauchsfilme 1896-1964, Zürich 2011, S. 147.

211 Sierek, Wie NinetTe Zu IHREM AuSGANG KAM, Lesemodus „Historischer Teil“, Kapitel „Werbefilm 1906-1918“, Anzeige „Erstes österreichisches konzessioniertes Reklamefilm-Institut“.

212 Fritz, Dokumentarfilme, Verzeichnis 1909-1914, Nr. 25, 29, 30, 32, 44-48. 
Kinematographentheater, so bot die „Sascha-Filmfabrik“ den Verleihern unter anderem Bilder Aus DEN DOLOMiten (1912), AN DEN UfERN DES GARDASEes (1912), DiE BuRg KREUZENSTEIN BEI WiEN (1912), AN DALMATIENS HeRRLiCHER KÜSTE (1912), ${ }^{213}$ BOZEN UND DER LuFTKURORT GRIES (1913), DuRCH DAS WILDROMANTISCHE EGGENTHAL (1913), Von Melk bis Krems (1913), Von Ragusa naCh Cattaro (1914), Mariazell Und UMgeBung (1914), Motive Aus MöDLING (1914) $)^{214}$ an.

Die österreichischen Produzenten hatten sich im Bereich der touristisch motivierten Filmerzeugung nachhaltig etabliert, auch die Gründung des ersten konzessionierten österreichischen „Reklame-Filminstituts“ wurde seitens der Fachpresse im Oktober 1913 verkündet. ${ }^{215}$ Unter zunehmend national anmutenden Tönen wuchs gleichzeitig der Wunsch nach „der kinematographischen Repräsentation des heimischen Terrains““. ${ }^{216}$ Trotzdem dominierten die internationalen Produktionsfirmen in allen Genres weiterhin den österreichisch-ungarischen Markt. Das sollte sich nach 1914 kriegsbedingt schließlich ändern.

\subsection{Wie NinetTe ZU IHRem Ausgang KAM - Der älteste überlieferte Konsumartikelwerbefilm österreichischer Provenienz}

Als erste kinematographische Werbefilmproduktion weltweit gilt der 1897 von Lavanchy Clarke, dem Vertreter eines englischen Waschmittelherstellers, bei den Brüdern Lumière in Auftrag gegebene Film SunLIGHT SEIFE. Der Reklamestreifen wurde anlässlich der Schweizer Landesausstellung in Genf von 70.000 Besuchern gesehen. Unter dem Titel WASHING DAYS IN SwITZERLAND fand der Film auch in New York sein Publikum. ${ }^{217}$ In Deutschland hatte die konsumzentrierte Filmpropaganda 1898 ihre Premiere. Der Filmpionier Oskar Messter produzierte unter der Prämisse BADE ZU HAUSE! seinen ersten Werbefilm. Angepriesen wurde eine „Wellenbadschaukel“ der Berliner Firma Moosdorf \& Hochhäusler. ${ }^{218}$

213 Österreichischer Komet, „Anzeige ,Sascha'-Filmfabrik“, Nr. 92, 17. Februar 1912, S. 23. Ebd., „Anzeige „Sascha'-Filmfabrik“, Nr. 90, 3. Februar 1912, S. 29. Ebd., „Anzeige „Sascha'-Filmfabrik“, Nr. 99, 6. April 1912, S. 31.

214 Fritz, Dokumentarfilme, Verzeichnis 1909-1914, Nr. 96, 125, 146, 147, 166.

215 Seinen Sitz hatte das „Reklame-Filminstitut“ im 7. Wiener Gemeindebezirk, Neubaugasse 11. Es verfolgte den Zweck, „mit Hilfe des Kinofilms nach amerikanischem und französischem Vorbild in den Kinotheatern die weitgehende Reklame für Handel, Industrie, Gewerbe, Fremdenverkehr sowie für das Ausstellungswesen zu machen“. Siehe: Österreichischer Komet, Nr. 177, 4. Oktober 1913, S. 37.

216 Vgl. Deeken, Geschichte und Ästhetik des Reisefilms, S. 313.

217 Bochsler/Derungs, Und führe uns in Versuchung, S. 45-46. Fleckenstein, Der französische Werbefilm, S. 127.

218 Messter, Oskar: Mein Weg mit dem Film, Berlin 1930, S. 131. Agde, Flimmernde Versprechen, S. 10 . 
In Österreich startete der „werbende Film“ vornehmlich im Bereich der Tourismusbranche. Die erste kinematographische Reklame für einen Konsumgüterartikel lässt sich für das Jahr 1913 belegen. WIE NINETTE ZU IHREM AUSGANG KAM wirbt für das Waschmittel Neubozon, das zu „den Spitzenreitern“ seiner Warenkategorie „zählte“ und von 1908 bis 1940 von der Unternehmerfamilie Neuber produziert wurde. ${ }^{219}$ Die Werbebotschaften „Nur mit Neubozon blendend weiße Wäsche!“ oder „Neubozon wäscht von selbst“ fanden vornehmlich auf Plakaten, Blechschildern, Postkarten oder in Anzeigen ihren Niederschlag. ${ }^{220}$ Vor dem Ersten Weltkrieg erprobte die Firmenleitung eine weitere Reklameform und beauftragte die „Sascha-Filmfabrik“ mit der Konzeption und Umsetzung eines Werbefilms. Eine Kopie des Streifens sowie das Originaldrehbuch finden sich in der Sammlung des Filmarchiv Austria. Dabei handelt es sich zudem um das älteste erhaltene Skript der österreichischen Filmhistorie.

WIE NinETTE ZU IHREM AUSGANG KAM weist die narrative Makrostruktur eines klassischen Waschmittelspots auf: Problemsituation, Konkretisierung des Problems, Empfehlung, Anwendung, Wirkungsdemonstration, Problemlösung, Bestätigung und Schlussappell. ${ }^{221}$ Die Erzählweise und die Überzeugungsstrategien basieren auf einer dissoziativen Dramaturgie: ${ }^{222}$ Verschmutze Wäsche wird als ein Problem erkannt, ein empfohlenes Produkt zur Anwendung gebracht und seine Wirkungsweise demonstriert. Die (hygienisch) saubere und farbechte Wäsche bestätigt den Erfolg des Waschmittels. Das Problem ist gelöst, ein eindringlicher Schlussappell preist den Verkaufsartikel nochmals an. Dabei werden „Alltagssituationen“ strapaziert, die „der potentiellen Käuferin“ (die Filme richten sich fast ausschließlich an ein weibliches Publikum) Nähe und Vertrautheit vermitteln sollen.

Der erste österreichische Konsumgüterwerbefilm folgt der Erzählform Slice-oflife. Das Setting gibt ein Motiv aus dem Arbeitsalltag wieder. Eine Waschküche wurde arrangiert. Ein Wäschekasten und ein Bügelbrett (links im Bild), Wäschekörbe und ein Waschtrog (rechts im Bild) sowie eine quer durch den Raum gespannte Wäscheleine legen die Funktion des Raums fest. Der mit Kreide an die Wand gemalte Satz „Der Franz, der Schuft“ wie auch die den Filmtitel erläuternde Bemerkung „Ein Scherz von Robert Reich“ verweisen auf den humoristischen Volksstückcharakter des Reklamestreifens. Auch das inszenierte „Bühnenbild“ mit einer mittig ausgerichteten Tür, die den Darstellerinnen und Darstellern Auftritt und Abgang ermöglichen, zeigen die Nähe zum Theater-Einakter deutlich auf.

219 Fischler, Gerhard/Kristen, Johanna: 1865-1990. 125 Jahre Wilhelm Neuber, Wien 1990, S. 14.

220 Vgl. dazu das Interview mit Gerhard Fischler (Urenkel des Firmengründers Franz Neuber): Sierek, WiE NinETTE ZU IHREM AUSGANG KAM, CD-Rom, Interviewsequenzen.

221 Vgl. dazu auch: Heiser, Drehbuch, S. 217.

$222 \mathrm{Zu}$ Dramatisierungsstrategien in der Werbung siehe hier: Schmidt/Spieß, Kommerzialisierung der Kommunikation, S. 156-158. 
Geachtet wurde auf eine möglichst ausgewogene Lichtsetzung sowie auf die Akzentuierung der Schwarz-Weiß-Kontraste: Bügelbrett, Waschbottich und Kleidungsstücke heben sich von der Umgebung hell ab. Ein einzelner, gezielt auf den Wäschekasten ausgerichteter Lichtreflex lässt auch diesem wichtigen Element des nachfolgenden Beweisführungsverfahrens erhöhte Aufmerksamkeit zukommen.

Von Beginn an erfolgt eine klare Typisierung der Figuren durch deren Kleidung und Gebärden. Ninette trägt weite Arbeitsaufmachung (Schürze, lockeres, per Kopftuch gebändigtes Haar) und ergeht sich in weinerlicher Verzweiflungsgestik. Die Hausherrin übt sich - in gutbürgerlichem Kostüm gekleidet - im drohenden Mienenspiel. Der uniformierte Soldat Jean zeugt von unerschütterlicher Heiterkeit und Optimismus. Der Kürze des Werbefilms entsprechend werden Charakterisierung und Funktion der handelnden Personen präzisiert und unmittelbar erfassbar.

Die Szenerie wird mit fest positionierter Kamera gefilmt, die im totalen Bildausschnitt die Schärfentiefe des Objektivs ausnutzt. Es liegt eine personale Erzählperspektive vor, der Point-of-view ist im Bewusstsein der Figuren angesiedelt. ${ }^{223}$ Der Film kommt ohne Zwischentitel aus.

Die dargelegte Lebenssituation der Zielgruppe vollzieht sich wie folgt: Die Hausherrin betritt die Waschküche, schreitet den Raum ab, während Ninette im Hintergrund entlangschleicht. Mit großen, aggressiven Gesten empört sich die Dienstgeberin über die unverrichtete Arbeit. Sie inspiziert die schmutzigen Kleidungsstücke, das Bügelbrett und öffnet den leeren Wäscheschrank. Das Dienstmädchen hält Arme und Hände näher am Körper, senkt den Kopf leicht, deutet Mutlosigkeit und Trotz an. Mit drohendem Zeigefinger geht die Frau des Hauses ab. Ninette ringt verzweifelt die Hände, einzeln hebt sie die verschmutzten Wäschestücke hoch, setzt sich auf den Wäschekorb und weint in theatralischer Manier. Die Tür öffnet sich, Jean betritt die Szenerie. Er grinst, klopft sich freudig auf den Schenkel, das Mädchen umarmt in herzlich. Ninette erklärt die aussichtslose Situation (ahmt das Reiben der Wäsche nach). Jean betrachtet die Kleidungsstücke, er hat einen Geistesblitz (tippt sich auf die Stirn). Die Hausherrin betritt den Raum, stellt sich zwischen die Liebenden und blickt erzürnt auf die noch immer schmutzige Wäsche. Der Soldat zieht sich zurück, verlässt die Waschküche. Unterdessen hält die Dame des Hauses Ninette eine Predigt, verstärkt das drohende Mienenspiel. Die Dienstgeberin lässt das Mädchen mit der Arbeit zurück. Letztere ist zusehends erzürnt, mit wütend erhobener Faust deutet sie der Herrin nach. Zornig wirft sie die Kleidungsstücke in den Waschtrog, beginnt mit verzagter Mimik die Wäsche zu säubern.

Jean kehrt zurück, er stellt demonstrativ ein Päckchen zur Schau. Der Soldat legt seinen Gürtel ab, öffnet das Paket und leert impulsiv den Inhalt in den Bottich. Das Pulver wirbelt hoch, Staubwolken erheben sich. Gemeinsam tunkt das Paar die

$223 \mathrm{Zu}$ den Point-of-view-Varianten siehe: Heiser, Drehbuch, S. 175-186. 
Wäsche eilig in die Lauge, wringt die einzelnen Stücke aus, um sie rasch und recht leger auf die Leine zu hängen. Slapstickartig laufen Ninette und Jean wiederholt mit der nassen Wäsche gegeneinander. Man scherzt und lacht. Nach einem Schnitt wird von der totalen zur halbtotalen Einstellung gewechselt. Jean bügelt die Tücher und legt sie fein säuberlich zusammen, während Ninette die Weißwäsche in den bereits gut gefüllten Schrank legt. Die Arbeit ist getan. Der Soldat klatscht frohgemut in die Hände, legt seinen Gürtel wieder an.

Jean kommuniziert nun direkt mit dem Publikum, die Handlungsachse ändert sich erstmals - statt nur zu beobachten, werden die Zuschauer einbezogen. Das Paar fällt sich glücklich in die Arme, Ninette wirft einen Blick zurück in die Kamera, die beiden verlassen den Raum. Kurz darauf betritt die Dienstgeberin die Waschküche. Sie sucht die Kleider im Korb, wendet sich zum Bügelbrett und steht schließlich erleichtert und sichtlich erfreut vor dem geöffneten, gefüllten Wäschekasten. Ninette und Jean schleichen indessen durch die Tür. Das Mädchen zeigt erklärend auf ihren Geliebten, der ein Werbeplakat der Firma Neubozon entrollt, auf dem eine schwarze, breit grinsende Waschfrau am Wäschetrog die Betrachter anblickt. Beide Frauen weisen auf die Affiche, die Hausherrin tippt sich lächelnd an die Stirn. Neuerlich wird das Publikum - nun im Zuge des Schlussappells - direkt adressiert. Das Endbild präsentiert das Werbeplakat in Nahaufnahme, der Kopf der Reklamefigur wurde durch den Ninettes ersetzt, die Frau blickt scherzend und nickend in die Kamera.

Ein Vergleich mit dem vorliegenden Ursprungsszenario macht Änderungen in der Umsetzung des Stoffes ersichtlich. Der realisierte Film weist einen weit weniger aggressiven Unterton auf. So stößt die Hausherrin Ninette weder in die Waschküche, noch geht sie wütend auf das Liebespaar los. Dafür bleiben sowohl ein Trinkgeld für das Mädchen als auch der vorgesehene Schlusstitel „Reichlich ist des Mädchens Lohn, wäscht sie nur mit Neubozon“ aus. ${ }^{224}$

Der mehrfache Einstellungswechsel, erste Versuche, sich einer einfachen Schnitttechnik zu bedienen sowie die offensichtlich beharrliche Anlehnung an eine Theaterinszenierung machen WIE NINETTE ZU IHREM AUSGANG KAM zu einem Paradebeispiel der Umbruchzeit zwischen 1900 und 1914. Das Kino befand sich in dieser Periode in einem klaren Spannungsfeld zwischen Attraktion und einsetzender Narration. ${ }^{225}$ Besonders interessant ist allerdings die Entwicklung der Figurenkonstellation. Über die Protagonistin, hier Ninette, findet der Zuschauer gewöhnlich den Zugang zur Geschichte. ${ }^{226}$ Ihr als Identifikationsfigur steht die Antagonistin (Hausherrin) in der Konfliktsituation gegenüber. Hier dynamisiert jedoch Jean den Verlauf der Handlung. Er ist der „Held“

224 Im Setting fehlt zudem eine zweite, im Skript angeführte, Tür. Siehe dazu: Filmarchiv Austria (FAA), Konvolut Wie NinETtE ZU IHREM AuSGAng KAM, Szenarium.

225 Vgl.: Sierek, WiE NinETTE ZU IHREM Ausgang KAM, Lesemodus „Theoretischer Teil“, Kapitel „Attraktion und Narration“.

226 Vgl. Heiser, Drehbuch, S. 146. 
des Plots und entwickelt sich von der Nebenfigur, als die er eingeführt wird, zum zweiten Protagonisten. Seine aktive Teilnahme an der (vor allem zu jener Zeit) weiblich konnotierten Arbeit wurde im damaligen kulturell-gesellschaftlichen Kontext durchaus als (wie im Untertitel eingeführter) „Scherz“ gedeutet. Zugleich treibt Jean das Geschehen an, ist für die Produkt- und Wirkungsdemonstration verantwortlich. Er verfügt über das Wissen, löst das Problem ${ }^{227}$ und verändert durch seine Tat die emotionale Stimmungslage der beiden Frauen, die sich seiner positiven Grundstimmung letztlich angleicht. Durch diese dominante, lenkende Position wird seine männlich uniformierte Kodifizierung wiederhergestellt.

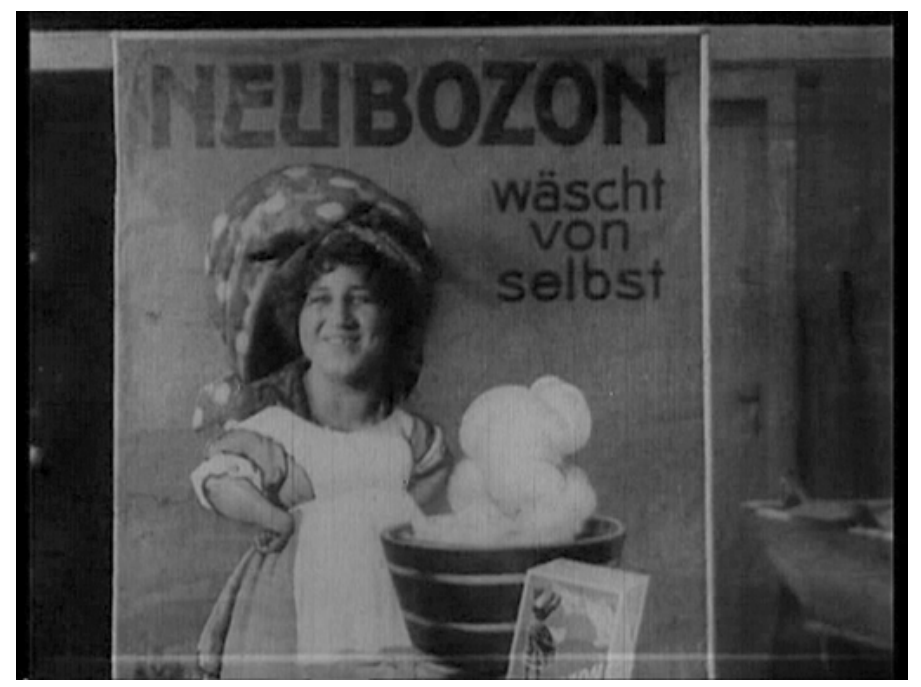

Abb. 2: Wie NinetTe ZU IHREM Ausgang KAM, A 1913.

Jean bestimmt auch die zusehends humoristische Tonalität des Films mit der implizierten Botschaft, dem Alltag stets etwas Positives abgewinnen zu können. Dabei tritt das beworbene Produkt beinahe völlig in den Hintergrund. Dass die Verwendung des Waschpulvers die Situationsveränderung (mit)bedingt, bleibt - aufgrund der hauptsächlich von Jean getragenen Humoreske - bis zum eingehenden Schlussappell letztlich ausgespart. Im Gesamten wird die Reklamebotschaft schließlich auf den Punkt gebracht: Neubozon vereinfacht das Wäschewaschen nicht nur, sondern

227 Damit steht Jean in der späteren Tradition des männlichen, allwissenden und beratenden Presenters, der eben auch in Haushaltsfragen fundierte Auskünfte und Tipps geben kann. Erinnert sei hier nur an Jan-Gert Hagemeyer, der als Persil-Mann jahrzehntelang über die Waschfunktion des Produkts per Werbespot informierte. 
macht die Hausarbeit zudem freudvoller, schneller und effizienter, was in der Folge auch mehr Freizeit in Aussicht stellt.

Ob und in welchem Ausmaß der Werbefilm den Kauf des Waschmittels beeinflusste, ist nicht zu eruieren. Belegbar ist jedoch der weitläufige Einsatz des neuen Reklamemittels. Ein Vertriebsplan des Unternehmens „Rudolf Ethofer \& Co“ weist Wien, Prag, Brünn, Lemberg, Klagenfurt, Linz, Innsbruck, Salzburg, Triest, Graz, Troppau, Zara, Bregenz, Sarajevo, Görz und Czernowitz als Aufführungsorte aus. ${ }^{228}$ An der Gestaltung des Werbe-Laufbilds waren mit Robert Reich als Drehbuchautor und Hans Otto Löwenstein als Regisseur zwei bedeutende Vertreter der österreichischen Filmbranche beteiligt. Reich bekleidete ab den 1920er-Jahren gewichtige Positionen in der heimischen Filmindustrie und sollte $\mathrm{zu}$ einem der wichtigsten Werbefilmproduzenten der Zwischenkriegszeit werden. ${ }^{229}$ Löwenstein gründete in den 1920er-Jahren die „Astoria“-, „Ottol“- und „Apollo“-Filmgesellschaften in Wien und war als Schauspieler, Drehbuchautor, Filmregisseur und -produzent tätig. Beide arbeiteten während des Ersten Weltkriegs aktiv für die k.k. Filmpropaganda des Kriegspressequartiers (KPQ). ${ }^{230}$

In den Jahren 1914 bis 1918 intensivierte sich der Einsatz neuer Propagandamedien. Auf allen Ebenen wurde für die Kriegsmaschinerie geworben. Die Laufbilder hatten $\mathrm{zu}$ überzeugen, mussten argumentativ sein, und deren Botschaft musste unmittelbar vom Publikum verstanden werden. Damit erfolgte nicht nur eine Abkehr von der „Ansicht“ hin zum narrativen Film, es wurden für die weitere Entwicklung und Akzeptanz des „werbenden Films“ wichtige Weichen gestellt.

228 FAA, Konvolut Wie NinetTe ZU IHREM AUSGANG KAM, Verzeichnis der für die Filmreklame angeschlossenen Kinos der Abteilung Film-Reklame der Unternehmung Rudolf Ethofer \& Co.

229 Siehe dazu das Unterkapitel „Robert Reich“.

230 Loacker/Prucha, Unerwünschtes Kino, S. 60. Loacker, Armin: Kino vor dem KZ. Österreichische Filmschaffende als NS-Opfer“, in: filmarchiv 7, 03/2003, S. 18. Sierek, WIE NINETTE ZU IHREM AUSGANG KAM, Lesemodus „Historischer Teil“, Kapitel „Hans Otto Löwenstein“. 